\title{
Caracterização da formação de cheias na bacia do rio Una em Pernambuco: simulação hidrológica-hidrodinâmica
}

\section{Characterization of flood formation in the Una river basin, Pernambuco: hydrological-hydrodynamic simulation}

\begin{abstract}
Alfredo Ribeiro Neto; José Almir Cirilo ${ }^{2}$ Carlos Eduardo de Oliveira Dantas ${ }^{3}$ e Edilson Raimundo Silva ${ }^{1}$
${ }^{1}$ Universidade Federal de Pernambuco - Centro de Tecnologia e Geociências, Dep. de Engenharia Civil, Lab. de Hidráulica, Recife/PE, Brasil alfredoribeiro@ufpe.br; edilsonrsilva@yahoo.com.br
\end{abstract}

${ }^{2}$ Universidade Federal de Pernambuco - Centro Acadêmico do Agreste, Caruaru/PE, Brasil almir.cirilo@gmail.com

${ }^{3}$ Serviço Geológico do Brasil, SUREG-RE, Recife/PE, Brasil

carlos.dantas@cprm.gov.br

\begin{abstract}
RESUMO
Em junho de 2010, ocorreu nos Estados de Pernambuco e Alagoas um evento bidrológico que causou severos danos às cidades localizadas nas margens dos principais rios da zona da Mata dos dois Estados. A bacia do rio Una foi uma das mais afetadas pela cheia. Com uma área de 6.704,0 k.m2, a bacia do rio Una está localizada na região da Mata Sul e Agreste do Estado de Pernambuco. Para reconstituir o evento de junho 2010, utilizou-se o modelo chuva-vazão HEC-HMS e o modelo bidrodinâmico HEC-RAS. A geometria do leito menor e maior do rio Una e seu afluente Pirangi foi determinada por meio de levantamento com técnica LiDAR (Light Detection and Ranging), que utiliza feixes de laser emitidos a partir de uma aeronave para escanear a área do rio $e$ adjacências. O objetivo do artigo é simular os eventos de cheia ocorridos na bacia hidrográfica do rio Una, observando os principais aspectos que influenciam a formação da cheia no rio e como isso influencia o processo de calibração dos modelos. Foram testados modos de calibração contínuo e por eventos, ambos de forma manual. Verificou-se que a simulação contínu inviabiliza o uso de um conjunto único de parâmetros para os períodos chuvoso e de estiagem. A melhor opção foi calibrar os modelos com dois eventos de cheia e verificar com outros seis eventos. Em seguida, reconstituiu-se o evento de 2010 para estimativa da vazão ocorrida, pois entende-se que esse foi o mais severo já ocorrido a despeito da falta de registro de vazão. O resultado pôde ser comparado apenas com a profundidade da água espacializada na cidade de Palmares. Observou-se superestimação da profundidade calculada pelo modelo HEC-RAS, mas uma boa aderência à abrangência da área inundada.
\end{abstract}

Palavras Chave: Cheias ribeirinhas. Eventos extremos. Bacia do rio Una. LiDAR

\begin{abstract}
:
In June 2010, a bydrological event caused severe damage to cities located along the banks of the main rivers in Zona da Mata in the States of Pernambuco and Alagoas. The Una River basin was one of the most affected by the flood. The Una River basin covers 6,704.0 km2 and is situated in the Zona da Mata and Agreste of Pernambuco State. To represent the event of June 2010, the rainfall-runoff model HEC-HMS and the hydrodynamic model HEC-RAS were used. The geometry of the minor and major riverbed of the Una River and its tributary Pirangi River was determined by mean of surveying using the LiDAR technique (Light Detection and Ranging), which employs laser beams emitted from an aircraft to scan the river area and its proximities. The objective of the paper is to simulate the flood events that occurred in the Una river basin, verifying the main aspects that influence the formation of the flood in the river and how this influences the calibration of the models. Continuous and event-based manual calibration was tested. The results showed that it is not possible to get a single set of parameters for wet and dry periods. The best option was to calibrate the models using two flood events and validate them with six other events. After the calibration, the 2010 event was simulated to estimate the peak flow. The 2010 flood can be considered the most severe event that has ever occurred despite the lack of streamflow records. The result could be compared only to the spatialized water depth at Palmares city. There was an overestimation of the water depth calculated by the HEC-RAS model, but there was also a good agreement with the extent of the flooded area.
\end{abstract}

Keywords: River floods. Extreme events. Una river basin. LiDAR 


\section{INTRODUÇÃO}

As inundações ribeirinhas caracterizam-se pela tomada da planície de inundação pela água das cheias. Várias cidades do Brasil foram criadas ou expandidas para as áreas da planície de inundação dos rios. O resultado da falta de planejamento é a ocorrência de grandes prejuízos para a população durante os eventos de cheias que extravasam a calha principal do rio. Diversas cidades da Zona da Mata dos Estados de Pernambuco e Alagoas têm sofrido com enchentes freqüentes ocorridas na última década com essa característica.

O enfrentamento desse problema deve passar pelo estudo das enchentes por meio de simulação matemática de modo a permitir o planejamento das intervenções como, por exemplo, zoneamento da ocupação do solo, previsão de cheias e análise do impacto da construção de reservatórios de controle de cheias (DE SILVA; WEERAKOON; HERATH, 2014). A combinação de modelos chuva-vazão e hidrodinâmico permite avaliar, por exemplo, a magnitude da redução da vazão após a construção de um reservatório de controle de cheias (GÜL; HARMANCIOGLU; GÜL, 2010) e delineamento de área inundada para eventos ocorridos e para eventos projetados com diferentes tempos de retorno (KNEBL et al., 2005; LASTRA et al., 2008).

Uma grande dificuldade na análise de eventos extremos de inundação refere-se à disponibilidade de dados de nível e profundidade da água, vazão e abrangência espacial da área inundada para calibração dos modelos, o que leva a maior incerteza nos resultados obtidos (SANYAL; CARBONNEAU; DENSMORE, 2013). O evento de junho de 2010, por exemplo, causou a destruição da estação fluviométrica de Palmares no rio Una, que impediu o registro do nível máximo da água e a correspondente vazão. Como alternativa, utilizaram-se os níveis levantados a partir das marcas deixadas pela água nas edificações na cidade de Palmares/PE.

Por outro lado, ferramentas modernas podem auxiliar na simulação de cheias como, por exemplo, Sistemas de Informações Geográficas (SIG), LiDAR (Light Detection and Ranging) e produtos de sensoriamento remoto (DANTAS, 2012, MENDES; CIRILO, 2013). Estes produtos fornecem informações valiosas para a representação precisa do relevo ao longo do rio que está sendo simulado, permitindo a caracterização da seção transversal para a modelagem hidrodinâmica e, posteriormente, determinação da profundidade da água na área atingida pela inundação (BALES et al., 2007; USGS, 2012).

O trabalho aqui apresentado está dividido em dois artigos. O objetivo deste primeiro artigo é simular os eventos de cheia ocorridos na bacia hidrográfica do rio Una no Estado de Pernambuco, Nordeste do Brasil, observando os principais aspectos que influenciam a formação da cheia no rio e como isso influencia o processo de calibração dos modelos. Um objetivo específico foi reconstituir o evento de cheia ocorrido em junho de 2010, em que não houve registro de vazão em decorrência da perda de estações de medição de vazão durante o evento.

O segundo artigo busca conhecer o padrão de ocorrência das inundações mais severas e, a partir desse padrão, estabelecer estratégias e cenários que permitam prever e acompanhar a evolução das inundações na bacia.

\section{METODOLOGIA}

\section{Área de Estudo}

A bacia hidrográfica do rio Una, com área de 6.704,0 $\mathrm{km}^{2}$ está localizada na região da Mata Sul e Agreste do Estado de Pernambuco-Brasil. A bacia hidrográfica do rio Una desenvolve-se no sentido oeste-leste com um comprimento total de seu curso principal de aproximadamente $255 \mathrm{~km}$. A parte alta da bacia está localizada na Mesorregião do Agreste Pernambucano, que possui característica climática intermediária entre a Zona da Mata e o Sertão semiárido (Figura 1). O comportamento hidrológico da bacia é caracterizado por precipitações elevadas nas porções média e baixa da bacia, enquanto no trecho alto as precipitações médias observadas tendem a reduzir-se (PERNAMBUCO, 2002):

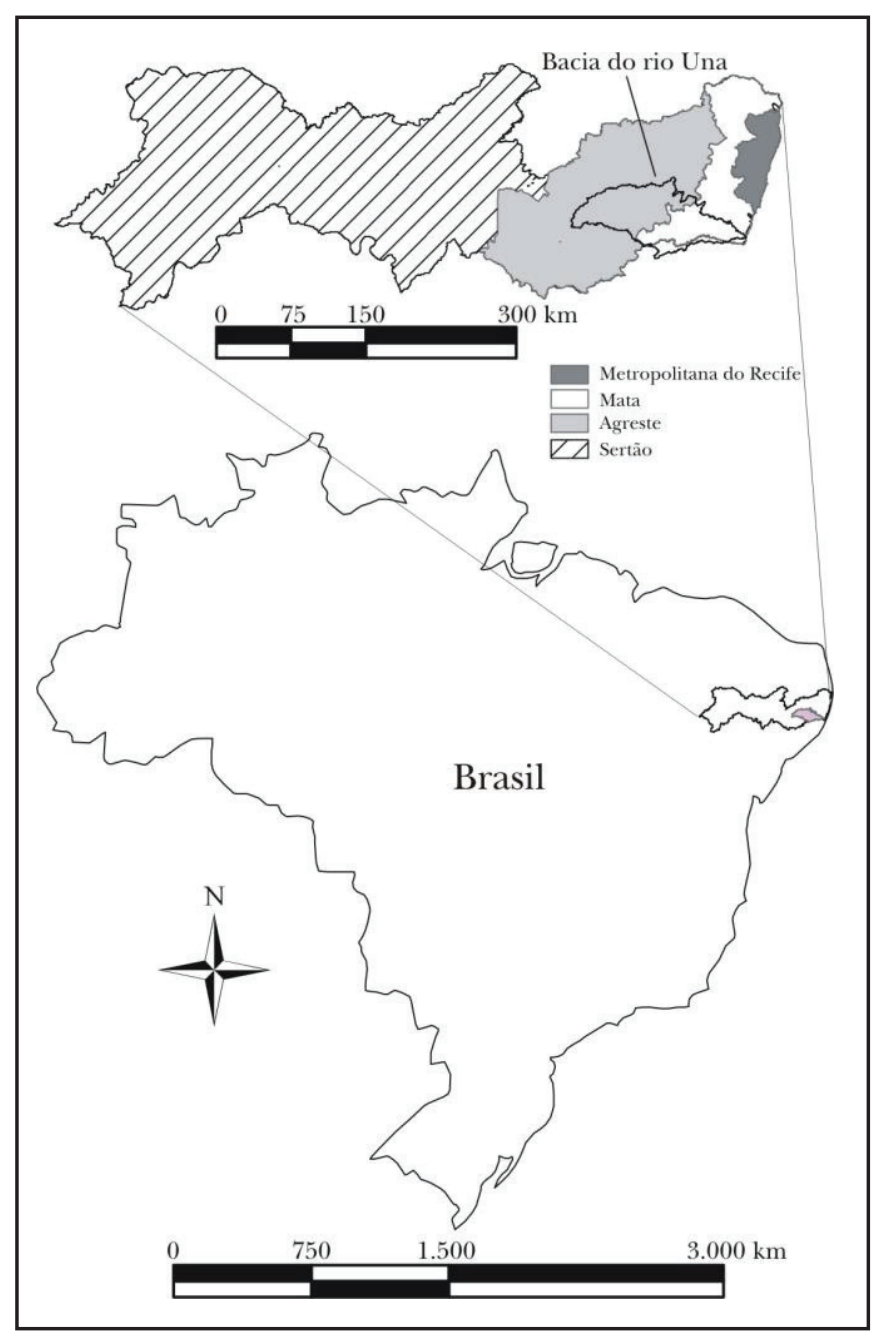

Figura 1 - Localização da bacia hidrográfica do rio Una

- O curso superior, com cerca de $2.900 \mathrm{~km}^{2}$ de área e precipitação média anual entre 600 e 800 mm, é caracterizado por escoamentos intermitentes anuais e grandes picos de enchentes de média recorrência. Março apresenta-se como o mês mais chuvoso;

- À medida que avança no sentido do litoral, o rio 
Una atravessa áreas da Mesorregião da Mata Pernambucana. O curso médio possui cerca de 2.100 $\mathrm{km}^{2}$ de área e precipitação média anual de $1.500 \mathrm{~mm}$. Nessa região, encontram-se os afluentes Pirangi e seu tributário Panelas. Esses rios são permanentes, apresentando picos de cheias significativos, considerando o padrão dos cursos d'água da região e maior repetição de vazões máximas, comparadas à sua bacia de contribuição, do que os observados no curso superior. Os maiores eventos de chuva ocorrem entre os meses de maio e agosto;

- No curso inferior, com cerca de $1.700 \mathrm{~km}^{2}$ de área e precipitação média anual de $2.000 \mathrm{~mm}$, encontramse os afluentes Santo Antônio e Jacuípe.

Verifica-se uma pequena defasagem no tempo entre as estações chuvosas das três zonas na bacia hidrográfica do rio Una. Enquanto essa estação finaliza em março/abril no curso superior, o seu início é em abril/maio no resto da bacia. No entanto, há anos em que podem ocorrer eventos de chuva provocados por sistemas atmosféricos como as ondas de leste em que toda a bacia é atingida. Associado a isso, a topografia do terreno, caracterizada por elevadas declividades, dará ao escoamento grande energia que provocará grandes danos nas cidades ribeirinhas.

\section{Dados Disponíveis}

Para a simulação hidrológica-hidrodinâmica, são necessárias séries de precipitação na bacia e vazão no rio Una e principais afluentes. A figura 2 mostra a distribuição espacial de 53 postos pluviométricos pertencentes às redes hidrometeorológicas da Agência Pernambucana de Água e Clima (APAC) e Agência Nacional de Águas (ANA). A figura 3 apresenta as estações fluviométricas e plataformas de coleta de dados (PCD). Uma parte das PCD's possui apenas pluviógrafos e outra parte possui pluviógrafos e linígrafos. As PCD’s são úteis para simulações com passo de tempo menor que o diário, particularmente importante para a bacia hidrográfica do rio Una, que possui pequeno tempo de resposta entre o evento de chuva e a chegada da onda de cheia nas cidades. As características das estações fluviométricas são descritas na tabela 1. A coluna com a vazão máxima diária para o tempo de retorno de 10 anos é apresentada porque esse é o valor utilizado como referência para a inclusão do evento no processo de calibração e verificação do modelo chuva-vazão.

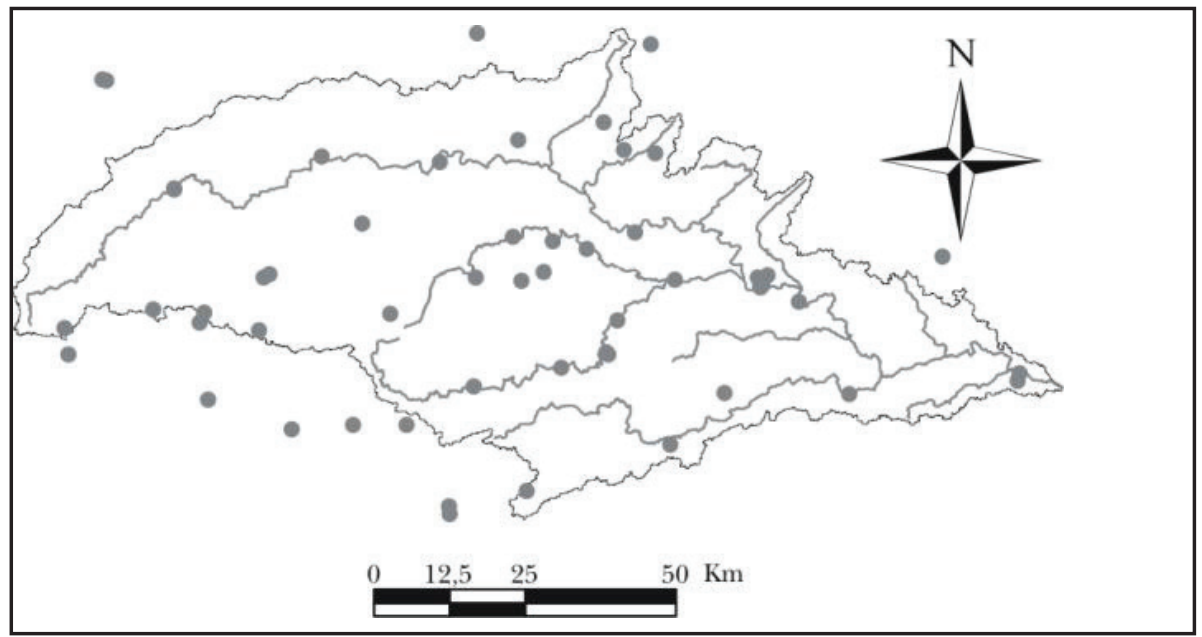

Figura 2 - Localização das estações pluviométricas

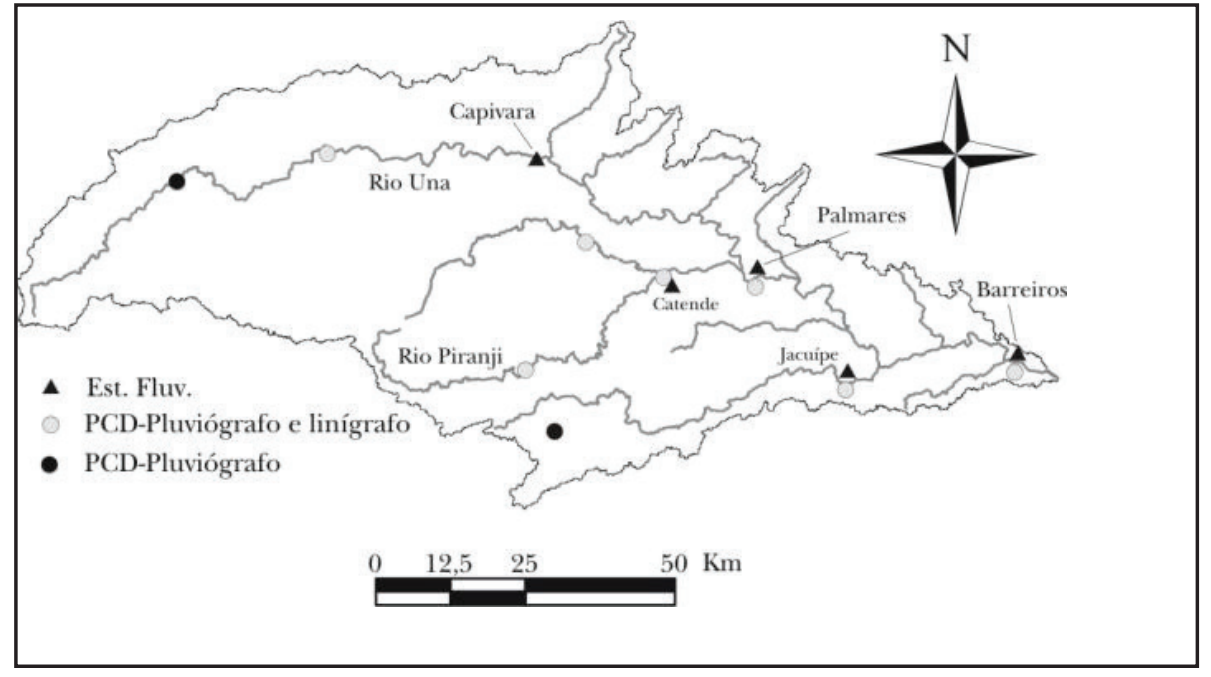

Figura 3 - Estações fluviométricas e pluviógrafos 
Tabela 1 - Características das estações fluviométricas

\begin{tabular}{c|c|c|c|c|c}
\hline Nome & Código ANA & Rio & Área $\left(\mathrm{km}^{2}\right)$ & Período & $\begin{array}{c}Q_{\max }(\operatorname{Tr}=10 \text { anos }) \\
\left(\mathrm{m}^{3} / \mathrm{s}\right)\end{array}$ \\
\hline Capivara & 39540000 & Una & $2.610,0$ & $1978-2011$ & 291,5 \\
\hline Catende & 39550000 & Pirangi & 718,0 & $1999-2011$ & 215,0 \\
\hline Palmares & 39560000 & Una & $4.910,0$ & $1967-2011$ & 636,3 \\
\hline Jacuípe & 39580000 & Jacuípe & 756,0 & $1989-2011$ & 324,0 \\
\hline Barreiros & 39590000 & Una & $6.550,0$ & $2002-2011$ & 893,0 \\
\hline
\end{tabular}

A representação da geometria do rio Una foi feita por meio de levantamento com técnica LiDAR (Light Detection and Ranging), que utiliza feixes de laser emitidos a partir de uma aeronave para escanear a área do rio e adjacências (aproximadamente $1 \mathrm{~km}$ de cada lado do rio) (Figura 4). O modelo digital do terreno (MDT) resultante possui resolução espacial de $0,5 \mathrm{~m}$ e precisão altimétrica de $15 \mathrm{~cm}$.

A modelagem hidrodinâmica necessita de uma condição de contorno a jusante do sistema. No presente caso, o limite de jusante será a foz do rio Una no oceano Atlântico. A alternativa para representar a condição de contorno de jusante foi a determinação da maré por meio do uso de constantes harmônicas da região próxima à foz do rio.

Figura 4 - Modelo digital do terreno LiDAR na confluência dos

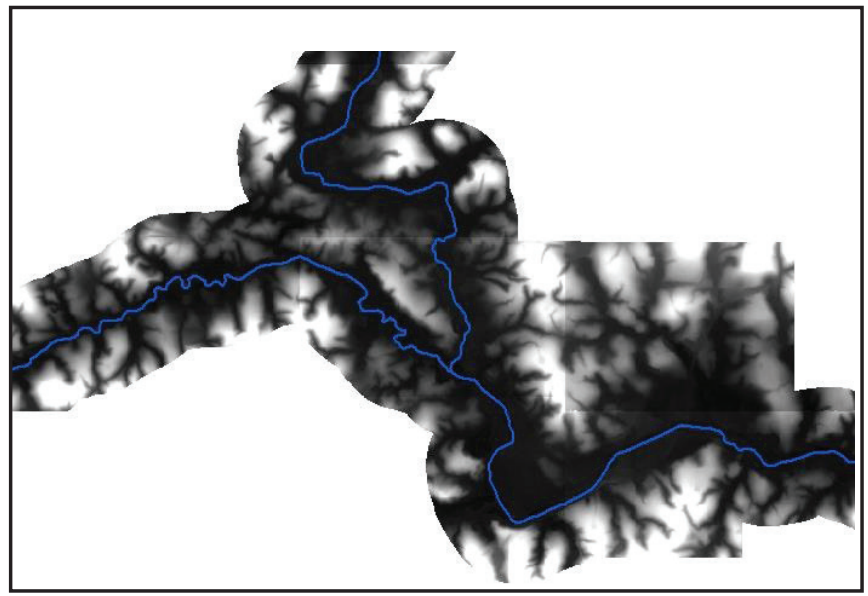

rios Una e Pirangi

\section{Modelos utilizados}

O HEC-HMS (Hydrologic Modeling System) é um modelo distribuído por sub-bacias desenvolvido pelo Hydrologic Engineering Center pertencente ao US Army Corps of Engineers. O modelo tem sido aplicado na solução de diversos problemas em uma grande variedade de bacias com diferentes características (DE SILVA; WEERAKOON; HERATH 2014, HABERLANDT; RADTKE, 2014, KNEBL et al., 2005).

O HEC-HMS é capaz de realizar simulações de eventos (de poucas horas ou dias) ou simulação contínua de longo período de modo que englobe períodos de cheia e de estiagem. Essa característica só é possível devido a um conjunto de modelos, formulações e equações que podem ser escolhidas para representar cada parte da fase terrestre do ciclo hidrológico (HEC-HMS, 2000): i) balanço hídrico na interface solo-vegeta- ção; ii) propagação do escoamento superficial; iii) propagação do escoamento subterrâneo; iv) propagação do escoamento na calha dos rios e reservatórios.

O modelo HEC-RAS (Hydrologic Engineering Center-River Analysis System) é capaz de simular escoamento unidimensional em regime permanente, regime transitório, transporte de sedimentos, leito móvel e modelagem da temperatura da água. Para a simulação em regime transitório, o modelo utiliza as equações completas de Saint Venant e o esquema de diferenças finitas implícito de quatro pontos (HEC-RAS, 2010). As equações de Saint Venant são constituídas pelas equações da continuidade e dinâmica:

onde A é a área da seção transversal, t o tempo, Q a vazão,

$$
\begin{gathered}
\frac{\partial \mathrm{A}}{\partial \mathrm{t}}+\frac{\partial \mathrm{Q}}{\partial \mathrm{x}}=\mathrm{q} \\
\frac{\partial \mathrm{v}}{\partial \mathrm{t}}+\mathrm{v} \frac{\partial \mathrm{v}}{\partial \mathrm{x}}+\mathrm{g} \frac{\partial \mathrm{y}}{\partial \mathrm{x}}+\mathrm{v} \frac{\mathrm{q}}{\mathrm{A}}=\mathrm{g}\left(\mathrm{S}_{0}-\mathrm{S}_{\mathrm{f}}\right)
\end{gathered}
$$

x a distância longitudinal no canal, q vazão de contribuição lateral, v a velocidade da água, g a aceleração da gravidade, y a profundidade da água, $\mathrm{S}_{0}$ é a declividade do fundo do rio e $\mathrm{S}_{\mathrm{f}}$ a declividade da linha de energia.

Além dessas duas equações, necessita-se de uma terceira que represente a resistência exercida pelo canal sobre o escoamento. A equação de resistência mais utilizada é a fórmula de Manning:

onde $\mathrm{R}$ é o raio hidráulico e n é o coeficiente de rugosidade

$$
\mathrm{v}=\frac{1}{\mathrm{n}} \cdot \mathrm{R}^{2 / 3} \cdot \mathrm{S}_{\mathrm{f}}^{1 / 2}
$$

de Manning.

Uma importante ferramenta disponível no modelo é a possibilidade de utilizar o Sistema de Informações Geográficas ArcGIS/ESRI para operações de pré e pós processamento dos dados de entrada e saída. O pré-processamento, a partir do uso de um modelo digital do terreno com informação do relevo, realiza a extração das seções transversais do rio e prepara um arquivo em formato compatível para ser importado pelo HEC-RAS. No sentido inverso, com os resultados da simulação hidrodinâmica, é possível realizar o mapeamento da planície de inundação no ArcGIS e, dessa forma, avaliar que partes das cidades são atingidas pelas cheias de diferentes magnitudes. 
Sistemas de rios com características diversas tem sido simulados com o modelo HEC-RAS (DOMENEGHETTI et al., 2014; KNEBL et al., 2005; SALEH et al., 2013).

\section{Simulações}

Inicialmente, a sequência de simulações teve três etapas. Primeiramente, o modelo chuva-vazão foi calibrado e verificado com simulações contínuas com passo de tempo diário. $\mathrm{Na}$ segunda etapa, o modelo hidrodinâmico foi calibrado e verificado por eventos utilizando como condições de contorno a vazão de saída do modelo chuva-vazão. Finalmente, na última etapa, com os modelos calibrados, simulou-se o evento de cheia de 2010.

A calibração e verificação do modelo chuva-vazão foram feitas nas estações da tabela 1. Procurou-se simular apenas o período correspondente à estação chuvosa de cada ano, pois o modelo apresentou dificuldade de simular a vazão, utilizando o mesmo conjunto de parâmetros, para os períodos úmido e seco. Uma vez que o interesse recai sobre os eventos de cheia, priorizou-se a calibração das vazões dos meses chuvosos dos anos que apresentassem vazão máxima com tempo de retorno superior a 10 anos (Tabela 1).

A calibração e verificação do modelo hidrodinâmico foi realizada tomando-se apenas os eventos de cheia, pois não é possível simular a propagação das vazões com o nível da água muito baixo. Isso acarreta instabilidades numéricas no modelo, causando interrupção da simulação. Novamente, tomou-se como referência o tempo de retorno de 10 anos. A estação fluviométrica de Palmares foi escolhida para a calibração porque possui a série mais extensa de vazões e sua localização é representativa da severidade dos eventos de cheia no rio Una.

A estratégia de calibração dos modelos teve que ser alterada porque a simulação dos eventos não apresentou bons resultados em decorrência de vazões subestimadas pelo modelo chuva-vazão. Optou-se, assim, pela calibração de forma simultânea dos dois modelos com simulações por evento.

\section{Calibração do Modelo Chuva-Vazão}

O modelo chuva-vazão HEC-HMS foi configurado utilizando-se os métodos Soil Moisture Accounting (SMA) para o balanço hídrico na bacia, hidrograma unitário SCS para a propagação na superfície da bacia, reservatório linear para a propagação da água subterrânea e Muskingum-Cunge para a propagação nos rios. O modelo SMA utiliza reservatórios para representar o armazenamento e o movimento da água na camada superficial do solo, na camada superior da zona saturada e na camada inferior da zona saturada.

O HEC-HMS é um modelo que pode funcionar de forma concentrada ou distribuído por sub-bacias. Para que a simulação tenha característica distribuída, é necessário discretizar a bacia em sub-bacias. A discretização da bacia hidrográfica do rio Una foi realizada com a extensão HEC-GeoHMS, que funciona em ambiente ArcGIS/ESRI, e resultou em um total de 51 sub-bacias (Figura 5). Além da operação de discretização, o HEC-GeoHMS pode ser utilizado, também, para a determinação de parâmetros do modelo com base em dados físicos como o modelo digital do terreno (MDT). Com o auxílio do MDT, calculou-se, por exemplo, os parâmetros do método de Muskingum-Cunge e o tempo de concentração das sub-bacias, informação útil para a determinação do parâmetro Lag Time do método do HU-SCS. Esse parâmetro é utilizado na determinação do hidrograma unitário e pode ser tomado como $60 \%$ do tempo de concentração na sub-bacia.

Os períodos de calibração e verificação são apresentados na tabela 2. Como não se utilizaram todos os dias do ano nas simulações, a tabela 2 apresenta, também, o total do número de dias simulados (n). Os parâmetros são atribuídos por sub-bacia e calibrados de forma manual. Os valores que se mostraram mais sensíveis sobre a vazão de saída foram infiltração máxima do solo (1,5 a 10,0 mm/h), capacidade de armazenamento do solo (40 a $500 \mathrm{~mm}$ ), área impermeável (5 a $20 \%$ ) e interceptação superficial $(7 \mathrm{~mm}))$. Os parâmetros foram calibrados manualmente procurando-se o melhor ajuste entre vazões observada e simulada. Maior atenção foi dada para a calibração da bacia contribuinte das estações fluviométricas de Capivara, no rio Una, Catende, no rio Pirangi, e Jacuípe no rio Jacuípe. As áreas

\section{RESULTADOS E DISCUSSÃO}

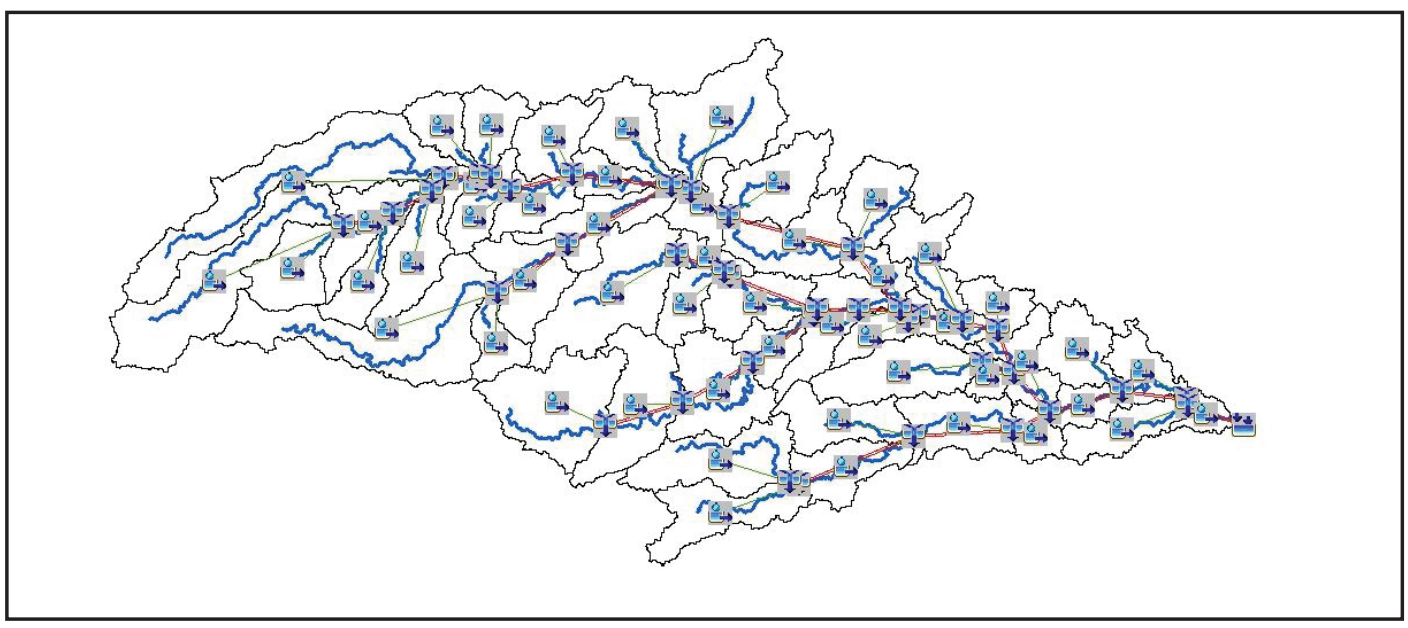

Figura 5 - Discretização da Bacia Hidrográfica do Rio Una no Modelo HEC-HMS 
Tabela 2 - Critérios de desempenho das simulações do modelo chuva-vazão: $\mathbf{n}=\mathbf{n}^{\circ}$ de dias de simulação, $\mathrm{m}=$ média das vazões, $\mathrm{DP}=$ desvio padrão das vazões, $\mathrm{CV}=$ coeficiente de variação, $\mathrm{R} 2$ = coeficiente de determinação

\begin{tabular}{c|c|c|c|c|c|c|c|c|c|c|c}
\hline & & \multicolumn{4}{|c|}{ Calibração } & \multicolumn{3}{c|}{ Vazão Calculada } & \multicolumn{3}{c}{ Vazão Observada } \\
\hline Estação & Período & $\mathrm{NS}$ & $\Delta \mathrm{V}$ & $\mathrm{R}^{2}$ & $\mathrm{n}$ & $\mathrm{m}$ & $\mathrm{DP}$ & $\mathrm{CV}$ & $\mathrm{m}$ & $\mathrm{DP}$ & $\mathrm{CV}$ \\
\hline Capivara & $1978-1990$ & 0,39 & 36,1 & 0,26 & 932 & 20,39 & 33,69 & 1,65 & 14,98 & 36,19 & 2,42 \\
\hline Catende & $1999-2007$ & 0,62 & $-21,7$ & 0,64 & 1057 & 11,20 & 12,66 & 1,13 & 14,52 & 21,12 & 1,45 \\
\hline Palmares & $1967-1990$ & 0,54 & 6,9 & 0,45 & 3610 & 53,48 & 48,75 & 0,91 & 53,40 & 69,45 & 1,30 \\
\hline Jacuípe & $1989-2000$ & 0,46 & $-14,4$ & 0,58 & 3610 & 13,66 & 19,93 & 1,46 & 18,74 & 22,07 & 1,18 \\
\hline Barreiros & $2003-2008$ & 0,53 & $-6,3$ & 0,46 & 861 & 77,5 & 56,75 & 0,73 & 82,79 & 85,07 & 1,03 \\
\hline & \multicolumn{9}{|c|}{ Verificação } & \multicolumn{3}{|c|}{ Vazão Calculada } & \multicolumn{2}{c}{ Vazão Observada } \\
\hline Estação & Período & $\mathrm{NS}$ & $\Delta \mathrm{V}$ & $\mathrm{R}^{2}$ & $\mathrm{n}$ & $\mathrm{m}$ & $\mathrm{DP}$ & $\mathrm{CV}$ & $\mathrm{m}$ & $\mathrm{DP}$ & $\mathrm{CV}$ \\
\hline Capivara & $2000-2011$ & 0,32 & 42,08 & 0,12 & 567 & 22,13 & 44,83 & 2,03 & 15,57 & 44,56 & 2,86 \\
\hline Catende & $2008-2011$ & 0,52 & 17,80 & 0,55 & 631 & 13,96 & 24,61 & 1,76 & 12,24 & 14,50 & 1,19 \\
\hline Palmares & $1991-2011$ & 0,59 & $-4,22$ & 0,52 & 631 & 44,02 & 37,36 & 0,85 & 45,96 & 41,96 & 0,91 \\
\hline Jacuípe & $2001-2011$ & 0,13 & $-14,03$ & 0,56 & 1078 & 20,05 & 41,19 & 2,05 & 23,33 & 28,21 & 1,21 \\
\hline Barreiros & $2009-2011$ & 0,20 & 3,54 & 0,50 & 462 & 95,56 & 118,71 & 1,24 & 92,29 & 92,36 & 1,00 \\
\hline
\end{tabular}

da bacia até a estação de Capivara mais os afluentes Pirangi e Jacuípe correspondem a $72 \%$ da bacia do rio Una e possuem papel importante na formação das cheias na bacia.

$$
\begin{array}{r}
\mathrm{NS}=1-\frac{\sum_{\mathrm{i}=1}^{\mathrm{n}}\left(\mathrm{Q}_{\mathrm{Calc}, \mathrm{i}}-\mathrm{Q}_{\mathrm{Obs}, \mathrm{i}}\right)^{2}}{\sum_{\mathrm{i}=1}^{\mathrm{n}}\left(\mathrm{Q}_{\mathrm{Obs}, \mathrm{i}}-\overline{\mathrm{Q}_{\mathrm{Obs}, \mathrm{i}}}\right)^{2}} \\
\Delta \mathrm{V}=\frac{\sum_{\mathrm{i}=1}^{\mathrm{n}} \mathrm{Q}_{\mathrm{Calc,i}}-\sum_{\mathrm{i}=1}^{\mathrm{n}} \mathrm{Q}_{\mathrm{Obs,i}, \mathrm{i}}}{\sum_{\mathrm{i}=1}^{\mathrm{n}} \mathrm{Q}_{\mathrm{Obs}, \mathrm{i}}} \cdot 100
\end{array}
$$

Foram utilizados os critérios de Nash Sutcliffe (NS) e erro de volume $(\Delta \mathrm{V})$ para avaliação do desempenho do processo de calibração e verificação.

onde

$\mathrm{Q}_{\text {Calc,i }}$ é a vazão calculada do dia i

$\mathrm{Q}_{\mathrm{Obs}, \mathrm{i}}$ é a vazão observada do dia $\mathrm{i}$

n é o número de dias do período de simulação

Os resultados da calibração do modelo HEC-HMS mostraram que, na maior parte do tempo da simulação, a vazão calculada acompanhou o momento de ocorrência dos picos de vazão medidos. A tabela 2 apresenta os valores de NS e $\Delta V$ na calibração e verificação nas cinco estações utilizadas. $O$ valor de n corresponde ao número de dias utilizados na simulação. Apresenta-se, ainda, a média (m), desvio padrão (DP), coeficiente de variação $(\mathrm{CV})$ e coeficiente de determinação $\left(\mathrm{R}^{2}\right)$. Comparando com outros estudos que utilizaram o modelo HEC-HMS, verifica-se um desempenho inferior na bacia hidrográfica do rio Una (DE SILVA; WEERAKOON; HERATH, 2014, HABERLANDT; RADTKE, 2014). Entretanto, em uma avaliação de três modelos hidrológicos na bacia hidrográfica do rio Una, Carvalho Neto, Ribeiro Neto e Montenegro (2014) observaram desempenho semelhante entre o modelo HEC-HMS e um modelo concentrado (MODHAC) e um modelo distribuído por células (MGB-IPH).

\section{Calibração do Modelo Hidrodinâmico}

Optou-se por uma estratégia diferente para a calibração do modelo hidrodinâmico. Foram simulados eventos ao invés de simulação contínua. Utilizaram-se os eventos listados na tabela 3 com o respectivo tempo de recorrência da vazão. Foi necessário simular eventos porque o modelo hidrodinâmico apresenta instabilidade numérica para escoamentos com baixa vazão.

Tabela 3 - Tempo de retorno da vazão registrada em Palmares dos eventos simulados

\begin{tabular}{c|c|c}
\hline Evento & Vazão máx. $\left(\mathrm{m}^{3} / \mathrm{s}\right)$ & $\operatorname{Tr}($ anos $)$ \\
\hline $\mathrm{Jul} / 1969$ & 605,0 & 8,8 \\
\hline $\mathrm{Jul} / 1970$ & 832,0 & 21,0 \\
\hline $\mathrm{Jul} / 1975$ & 1184,0 & 67,5 \\
\hline $\mathrm{Jul} / 1977$ & 613,0 & 9,1 \\
\hline $\mathrm{Jul} / 1988$ & 592,0 & 8,4 \\
\hline $\mathrm{Jul} / 1989$ & 661,0 & 11,0 \\
\hline Ago/2000 & $1.134,1$ & 57,8 \\
\hline Jun/2005 & 630,0 & 9,8 \\
\hline
\end{tabular}

O trecho simulado com o modelo HEC-RAS estendese da estação fluviométrica Capivara até a foz do rio no oceano Atlântico, incluindo o afluente rio Pirangi (Figura 3). Todo o processo de pré-processamento da geometria do rio Una foi realizado com o uso da extensão HEC-GeoRAS. Nessa etapa, constroem-se as linhas que representarão o eixo do rio, as margens e as seções transversais de onde se retiram os valores característicos de área, perímetro, raio hidráulico e declividade para a resolução das equações de Saint Venant. A figura 6 mostra um trecho do rio Una com os elementos geométricos representativos do sistema modelado. O espaçamento entre seções foi de $1 \mathrm{~km}$ nas áreas fora das cidades e 100 metros nas cidades para permitir um melhor detalhamento dos resultados e, consequentemente, do mapeamento das áreas de inundação.

Uma dificuldade encontrada diz respeito à representação deficiente do leito principal (ou leito menor) em algumas seções do rio. Isso ocorre porque o feixe de laser do levantamento LiDAR não consegue detectar o fundo do rio quando o mesmo 


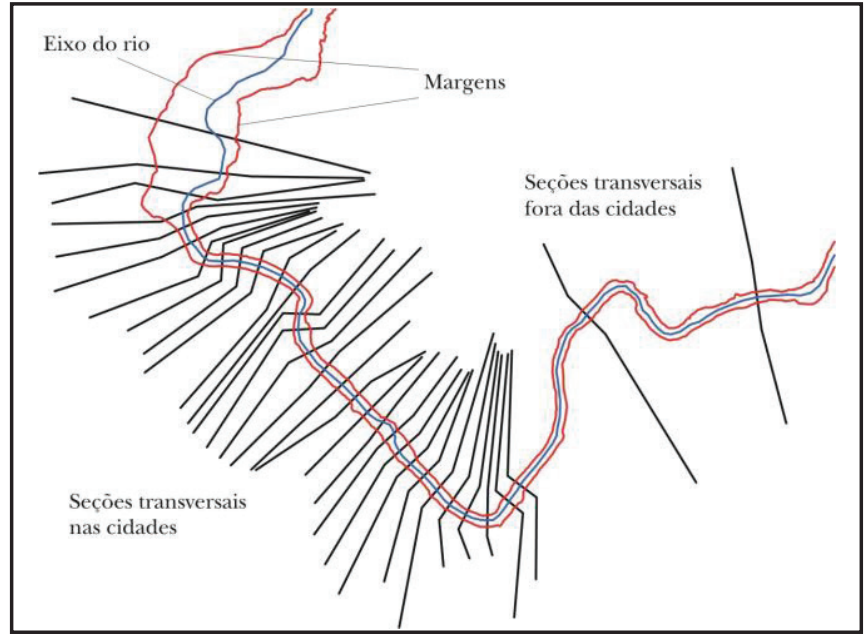

Figura 6 - Elementos geométricos representativos do sistema modelado

está submerso. O resultado é o aparecimento de instabilidades numéricas na resolução das equações de Saint Venant quando o nível da água está muito baixo. A solução desse problema foi a criação de um canal artificial no leito do rio por meio da ferramenta "Pilot Channel" do HEC-RAS. As dimensões do canal artificial foram largura de 1 metro e profundidade de 3 metros (Figura 7). Com isso, garante-se que sempre haverá uma lâmina d'água no fundo do rio, que permitirá a resolução das equações sem instabilidades numéricas.

O modelo HEC-RAS permite que se representem áreas de armazenamento localizadas ao longo das margens do rio. Essa opção de representação foi utilizada em todo o percurso simulado. Nas áreas de armazenamento, admite-se que não há escoamento da água, o que significa, em outras palavras, que se aplica apenas a equação da continuidade. $\mathrm{Na}$ área central, que representa o leito do rio, aplicam-se as equações da continuidade e dinâmica. Os valores do coeficiente de Manning foram calibrados visando, principalmente, evitar problemas com instabilidade numérica. Dessa forma, o valor mais apropriado foi 0,055 para o canal principal e para a planície de inundação adjacente ao rio conforme mostrado na figura 7 .

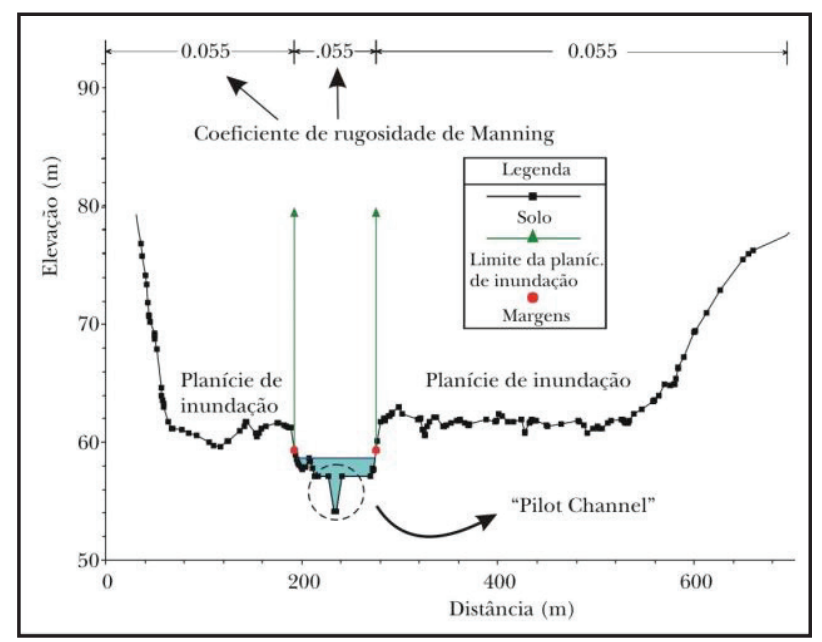

Figura 7 - Detalhes das seções transversais no modelo hidrodinâmico
A integração dos modelos HEC-HMS e HEC-RAS foi realizada por meio do sistema de armazenamento de dados gerados pelos programas computacionais do HEC. O sistema denominado Hydrologic Engineering Center Data Storage System (HEC-DSS) foi concebido para armazenar e recuperar dados científicos tipicamente sequenciais (como as séries de vazão em rios). Dessa forma, a vazão gerada pelo HEC-HMS é armazenada em um arquivo .dss, que, posteriormente, pode ser lido pelo HEC-RAS para a entrada das mesmas vazões nas condições de contorno do modelo hidrodinâmico. Foi utilizada a vazão gerada pelo HEC-HMS a montante da estação de Capivara e as vazões dos afluentes que deságuam ao longo dos rios Piranji e Una.

Ao se utilizar os resultados do HEC-HMS como entrada no HEC-RAS, verificou-se acentuada subestimação da vazão máxima do evento. Isso, provavelmente, ocorreu porque os parâmetros do modelo chuva-vazão foram ajustados para todo o período da estação chuvosa. Isso indica que, não apenas é necessário calibração com a vazão do período chuvoso, mas, preferencialmente, vazão do evento de cheia. Optou-se por modificar os valores dos parâmetros do modelo HEC-HMS para melhor ajuste do modelo hidrodinâmico. São eles a infiltração máxima $(1,5 \mathrm{~mm} / \mathrm{h}$ na parte superior e $2,0 \mathrm{~mm} / \mathrm{h}$ no médio e baixo curso), capacidade de armazenamento do solo (40 mm e $50 \mathrm{~mm}$ ), área impermeável (15\% e 20\%) e interceptação superficial $(0,0 \mathrm{~mm}$ para toda a bacia). Foram utilizados, na avaliação do modelo, os critérios do coeficiente Nash-Sutcliffe, erro de volume e erro percentual da vazão de pico (EQP) (Tabela 4). A calibração dos dois modelos de forma integrada foi realizada com os eventos de 2000 e 2005. Os demais eventos da tabela 3 foram utilizados para verificação.

Admitindo como satisfatórios os valores maiores que 0,50 para NS e menores que $25,0 \%$ para o valor absoluto de $\Delta V$ e EQP (MORIASI et al., 2007), verifica-se que os critérios ficaram dentro de uma faixa aceitável para cinco dos oito eventos simulados $(1975,1977,1988,2000$ e 2005). O tempo de pico da vazão máxima calculada foi igual ao observado para todos os eventos com exceção dos anos 1975 e 1989.

Moriasi et al. (2007) destacam que os limites aceitáveis dos critérios de avaliação da calibração de modelos hidrológicos devem ser menos exigentes para simulações com passo de tempo diário. A figura 8 mostra as vazões observada e calculada para todos os eventos da calibração e verificação com os respectivos valores do coeficiente de Nash-Sutcliffe. Observa-se que, apesar do desempenho ruim de três eventos da verificação, o valor global de NS enquadra-se como satisfatório.

Tabela 4 - Desempenho do modelo hidrodinâmico

\begin{tabular}{c|c|c|c}
\hline Evento & $\mathrm{NS}$ & $\Delta \mathrm{V}(\%)$ & $\mathrm{EQP}(\%)$ \\
\hline Jul/1969 & 0,1525 & $-30,01$ & $-54,46$ \\
\hline Jul/1970 & $-0,3519$ & 96,74 & 46,87 \\
\hline Jul/1975 & 0,7155 & $-25,94$ & $-14,10$ \\
\hline Jul/1977 & 0,5151 & $-17,39$ & $-26,52$ \\
\hline Jul/1988 & 0,8557 & 11,68 & $-6,25$ \\
\hline Jul/1989 & $-0,3142$ & $-15,65$ & $-57,42$ \\
\hline Ago/2000 & 0,6153 & 10,41 & $-8,31$ \\
\hline Jun/2005 & 0,5663 & 14,99 & $-23,73$ \\
\hline
\end{tabular}




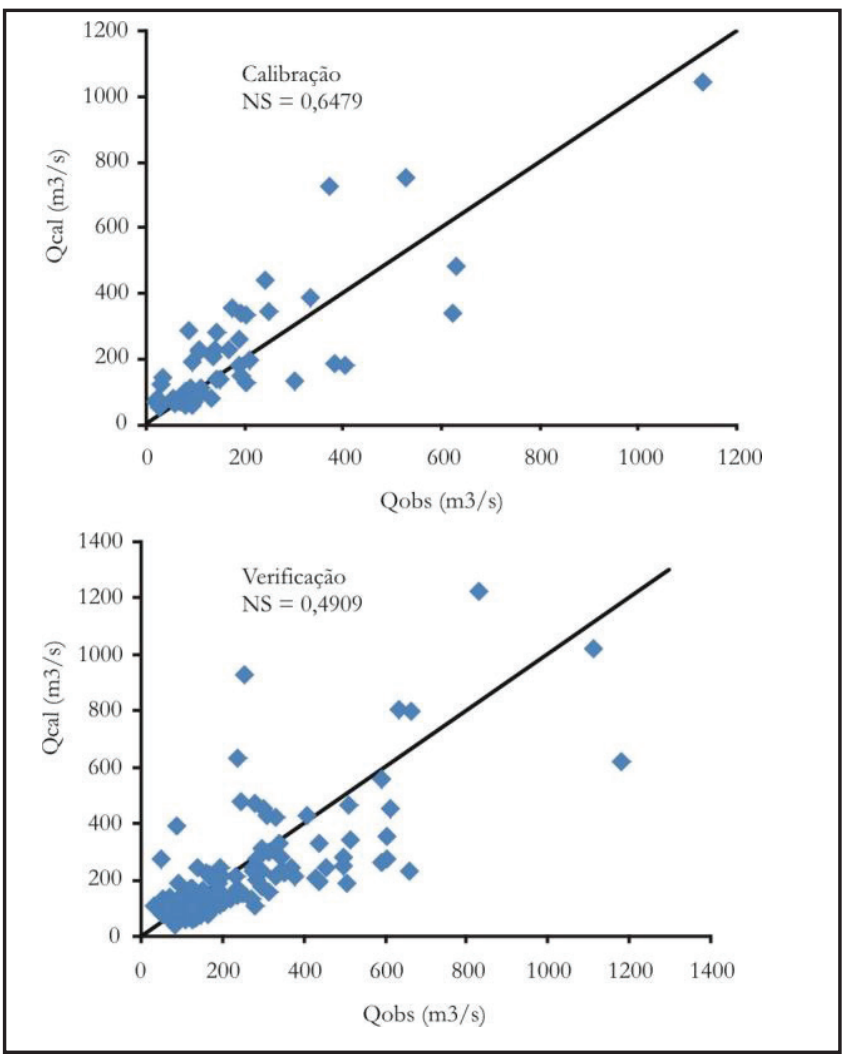

Figura 8 - Desempenho médio dos eventos da calibração e verificação do modelo hidrodinâmico

\section{Simulação do Evento de 2010}

Em junho de 2010, uma sequência de eventos nas bacias dos rios Capibaribe, Sirinhaém, Una, Mundaú e Paraíba, nos Estados de Pernambuco e Alagoas, resultaram em enchentes de grandes proporções. O primeiro evento ocorreu no período de 17 a 19 e o segundo nos dias 27 e 28 de junho.

O total de chuva em Palmares entre os dias 17 e 19 foi de $354,5 \mathrm{~mm}$, que equivalem a $43 \%$ acima da média histórica de todo o mês de junho. No município de Catende, foram registradas precipitações diárias totais de 55,122 e 130 milímetros respectivamente nos dias 17 a 19 . O total de chuva acumulado nestes três dias superou em mais de $30 \%$ o esperado para todo o mês de junho.

A partir da precipitação registrada nos postos pluviométricos, realizou-se a simulação do evento de junho de 2010 com os modelos calibrados. Em virtude da dimensão do evento, nenhuma estação fluviométrica localizada no rio Una registrou a sequência de níveis e vazões atingidas. No caso da estação de Palmares, houve, inclusive, a perda total das réguas e a Referência de Nível (RN).

Uma informação disponível que pode ser utilizada para a verificação do resultado dos modelos é o levantamento de marcas de cheia na cidade de Palmares realizado pelo Corpo de Bombeiros Militar de Pernambuco após o evento. Em 180 pontos ao longo da cidade, foram obtidas as coordenadas e a profundidade da marca até o solo. Este levantamento foi inserido em ambiente SIG e, por meio do MDT LiDAR de Palmares, os pontos do levantamento passaram a ter cotas das marcas de cheia em vez de alturas em relação ao terreno, gerando-se uma grade regular de pontos, um raster, de mesmo espaçamento do MDT LiDAR, através do interpolador IDW (Inverse Distance Weighted). O IDW foi escolhido por ser um interpolador exato, ou seja, nos locais em que o grid coincide com os pontos levantados, as cotas calculadas são iguais aos registros de campo, gerando superfícies mais suaves apesar da não adoção de fator de suavização.

A profundidade dos pontos medidos variou de 0,2 a 9,5 metros. A comparação dos valores simulados com os medidos foi feita subtraindo-se os dois valores e tomando-se o módulo. A figura 9 mostra a distribuição espacial da diferença absoluta da profundidade da água na área da cidade de Palmares atingida pela inundação. $\mathrm{O}$ valor médio da diferença foi de 4,3 metros e o desvio padrão igual a 0,95 metros.

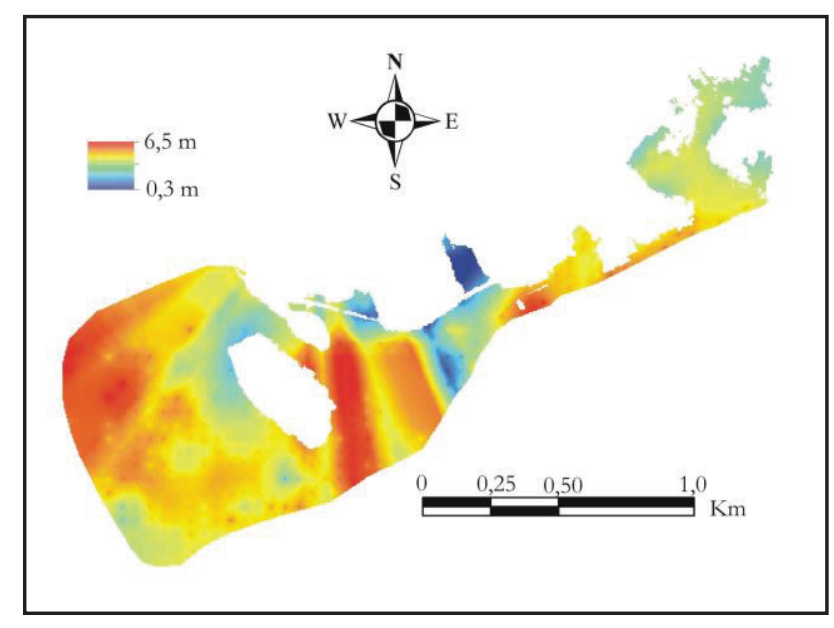

Figura 9 - Distribuição espacial da diferença absoluta da profundidade medida e profundidade calculada pelo modelo HEC-RAS na cidade de Palmares no evento de junho de 2010

Para avaliação da vazão simulada, tomou-se como base a estimativa de medição de vazão realizada por Engenheiros da Secretaria de Recursos Hídricos e Energéticos de Pernambuco durante o evento de cheia em Palmares. Com o uso de um flutuador, determinou-se a velocidade da água e a vazão estimada foi de $1.800,0 \mathrm{~m}^{3} / \mathrm{s}$ (SRHE, 2010). O valor da vazão máxima simulada com os modelos HEC-HMS e HEC-RAS foi de 2.232,0 $\mathrm{m}^{3} / \mathrm{s}$. Apesar de não se ter garantia de que a vazão estimada com o flutuador represente o pico do evento, a medição é uma boa referência para comparação como a vazão calculada. $\mathrm{Na}$ avaliação de simulações de um modelo hidrodinâmico, é importante que se verifique a coerência da relação entre o nível da água e a vazão correspondente. Ao se comparar o nível medido com o simulado e a vazão estimada com a simulada em Palmares, tem-se uma forma de se verificar se a relação nível-vazão está coerente.

$\mathrm{Na}$ estação fluviométrica de Barreiros, a vazão máxima simulada com os modelos foi de $2.420,0 \mathrm{~m}^{3} / \mathrm{s}$ e, na foz do rio Una, a vazão chegou a $2.313,0 \mathrm{~m}^{3} / \mathrm{s}$. Utilizando 42 anos de dados de vazão em Palmares, ajustou-se a distribuição Generalizada de Valores Extremos (GEV) para determinação do tempo de retorno das vazões máximas. Admitindo-se que a estimativa de quantil máxima recomendada é de três vezes o tamanho 
da série, não é possível afirmar com exatidão qual o tempo de retorno associado ao evento de 2010. Pode-se apenas afirmar que, considerando que a vazão para o tempo de retorno de 150 anos é de $1.461,41 \mathrm{~m}^{3}$ / s, o tempo de retorno do evento de 2010 pode chegar a 200 anos.

\section{CONCLUSÕES}

O aumento da freqüência de eventos extremos na última década nos rios da zona da Mata do Estado de Pernambuco tem causado grandes prejuízos para a população e exige o estudo e desenvolvimento de metodologias que permitam a análise das cheias e a sua previsão. A integração dos modelos HEC-HMS e HEC-RAS permite que, a partir dos dados de precipitação medidos ou previstos, realize-se a propagação da vazão nos principais rios da região. Os resultados obtidos com o modelo chuva-vazão HEC-HMS indicam que a parametrização do modelo é capaz de representar os processos hidrológicos na bacia hidrográfica do rio Una e, assim, fornecer os valores de vazão necessários para a representação das condições de contorno do modelo hidrodinâmico. As características da bacia hidrográfica do rio Una (elevada declividade e clima heterogêneo) exigem o uso de modelo chuva-vazão, pois apenas a propagação da água presente no canal não é suficiente. A formação da cheia se dá de forma muito rápida e a modelagem chuva-vazão concede um ganho de antecedência na previsão.

Na calibração dos modelos, testaram-se estratégias que melhor respondessem às características da bacia. Inicialmente, procurou-se calibrar o modelo HEC-HMS utilizando-se os períodos chuvosos de cada ano. Ao se utilizar as vazões calculadas no HEC-HMS como entrada do modelo HEC-RAS, observou-se que, apesar do desempenho satisfatório do modelo chuva-vazão, o resultado não era bom o suficiente para simular os grandes eventos de cheia. Isso levou à uma segunda estratégia de calibração em que se utilizou simulação por evento. A calibração, nesse caso, foi feita de forma integrada onde os parâmetros dos dois modelos foram ajustados simultaneamente. As tentativas de calibração dos modelos mostraram que a simulação contínua é inviável para a bacia hidrográfica do rio Una, pois não foi possível identificar um conjunto de parâmetros único que permita simular de forma igualmente satisfatória as vazões baixas e vazões extremas de cheias.

$\mathrm{Na}$ simulação hidrodinâmica, fez-se uso de dados altimétricos obtidos por meio da técnica de sensoriamento remoto LiDAR. O elevado nível de precisão dessa técnica é útil para o mapeamento da planície de inundação nas cidades atingidas pelas enchentes. Um problema encontrado refere-se à má representação da geometria do leito do rio que pode resultar em instabilidade numérica, principalmente, nos intervalos de tempo em que o nível da água está baixo, ou seja, pequena lâmina d'água. As instabilidades foram sanadas com a inclusão de um pequeno canal no leito do rio utilizando-se ferramenta disponível no próprio HEC-RAS.

Além da comparação com as vazões da estação de Palmares, realizou-se avaliação do modelo hidrodinâmico com os níveis da água medidos após o evento de junho de 2010. O modelo apresentou superestimação da profundidade da água por um lado, mas, por outro lado, a abrangência da área inundada ficou próxima da medida em campo. A simulação do evento de 2010 permitiu reconstituir as vazões nas estações que foram destruídas pela cheia. A vazão em Palmares, de acordo com o resultado da simulação, chegou a $2.232,0 \mathrm{~m}^{3} / \mathrm{s}$, que pode corresponder a um tempo de retorno de aproximadamente 200 anos.

Os modelos apresentados estão hoje integrados a um Sistema de Suporte à Decisão aplicado à previsão e alerta de cheias no rio Una, sendo refinados para incorporarem a previsão das chuvas. De posse da previsão de chuva na bacia, será possível determinar a vazão, o nível da água e as áreas atingidas nas cidades localizadas nas margens do rio Una, permitindo a mobilização com antecedência para a assistência à população. Além disso, a integração dos modelos permite a avaliação de ações propostas para a redução dos impactos das cheias na bacia hidrográfica do rio Una como, por exemplo, construção de reservatórios, obras de dragagem e zoneamento da planície de inundação.

\section{AGRADECIMENTOS}

Os autores agradecem ao Conselho Nacional de Desenvolvimento Científico e Tecnológico (CNPq), à Secreta-ria de Recursos Hídricos e Energéticos e ao Corpo de Bombeiros do Governo do Estado de Pernambuco, assim como ao Serviço Geológico do Brasil (CPRM), pelo apoio ao desenvolvimento da pesquisa. Este artigo é uma contribuição da Rede Brasileira de Pesquisas sobre Mudanças Climáticas Globais, convênio FINEP/Rede CLIMA 01.08.0405.01.

\section{REFERÊNCIAS}

BALES, J. D.; WAGNER, C. R.; TIGHE, K. C.; TERZIOTTTI, S. LiDAR-Derived Flood-Inundation Maps for Real-Time Flood-Mapping Applications, Tar River Basin, North Carolina. Reston, Virginia: U.S. Geological Survey, 2007. (Scientific Investigations Report, 2007-5032).

CARVAlHO NETO, J. G.; RIBEIRO NETO, A.; MONTENEGRO, S. M. G. L. Assessment of RainfallRunoff Models for Flood River Extreme Event Simulations. In: INTERNATIONAL CONFERENCE ON FLOOD MANAGEMENT, 6., 2014, São Paulo. Anais... São Paulo; [s.n.], 2014.

DANTAS, C. E. O. Previsão e Controle de Inundações em Meio Urbano com Suporte de Informacõos Espaciais de Alta Resolução. 2012. Tese (Doutorado em Engenharia Civil) - Universidade Federal de Pernambuco, 2012.

DE SILVA, M. M. G. T.; WEERAKOON, S. B.; HERATH, S. Modeling of Event and Continuous Flow Hydrographs with HEC-HMS: Case Study in the Kelani River Basin, Sri Lanka. Journal of Hydrologic Engineering, v. 19, n. 4, p. 800-806, Apr. 2014. 
DOMENEGHETTI, A.; TARPANELLI, A.; BROCCA, L.; BARBETTA, S.; MORAMARCO, T.; CASTELLARIN, A.; BRATH, A. The use of remote sensing-derived water surface data for hydraulic model calibration. Remote Sensing of Environment, v. 149, p. 130-141, June 2014.

GÜL, G. O.; HARMANCIOGLU, N.; GÜL, A. A combined hydrologic and hydraulic modeling approach for testing efficiency of structural flood control measures. Natural Hazards, v. 54, n. 2, p. 245-260, Aug. 2010.

HABERLANDT, U.; RADTKE, I. Hydrological model calibration for derived flood frequency analysis using stochastic rainfall and probability distributions of peak flows. Hydrology and Earth System Sciences, v. 18, 353-365, Jan. 2014.

HEC-RAS - River Analysis System. User's Manual. Davis, USA: US Army Corps of Engineers, 2010.

HEC-HMS - Hydrologic Modeling System. Technical Reference Manual. Davis, USA: US Army Corps of Engineers, 2000.

KNEBL, M. R.; YANGA, Z. L.; HUTCHISONB, K.; MAIDMENT, D. R. Regional scale flood modeling using NEXRAD rainfall, GIS, and HEC-HMS/RAS: a case study for the San Antonio River Basin Summer 2002 storm event. Journal of Environmental Management, v. 75, p. 325-336, 2005.

LASTRA, J.; FERNANDEZ, E.; DIEZ-HERRERO, A.; MARQUINEZ, J. Flood hazard delineation combining geomorphological and hydrological methods: an example in the Northern Iberian Peninsula. Natural Hazards, v. 45, n. 2, p. 277-293, May 2008.

MENDES, C. A. B.; CIRILO, J. A. Geoprocessamento em Recursos Hidricos. Princípios, Integração e Aplicação. 2. ed. Porto alegre: Associação Brasileira de Recursos Hídricos, 2013.

MORIASI, D. N.; ARNOLD, J. G.; VAN LIEW, M. W.; BINGNER, R. L.; HARMEL, R. D.; VEITH, T. L. Model Evaluation Guidelines for Systematic Quantification of Accuracy in Watershed Simulations. American Society of Agricultural and Biological Engineers, v. 50, n. 3, p. 885-900, 2007.

PERNAMBUCO. Relatório Técnico Preliminar (Anteprojeto) de 03 (Três) Barramentos para o Controle de Cheias na Bacia do Rio UnaPE (Sintese). [S.1.]: Téchne - Engenheiros Consultores, 2002.

SALEH, F.; DUCHARNE, A.; FLIPO, N.; OUDIN, L.; LEDOUX, E. Impact of river bed morphology on discharge and water levels simulated by a 1D Saint-Venant hydraulic model at regional scale. Journal of Hydrology, v. 476, 169-177, Jan. 2013.

SANYAL, J.; CARBONNEAU, P.; DENSMORE, A. L. Hydraulic routing of extreme floods in a large ungauged river and the estimation of associated uncertainties: a case study of the Damodar River, India. Natural Hazards, v. 66, n. 2, p. 1153-
1177, Mar. 2013.

SRHE - Secretaria de Recursos Hídricos e Energéticos. Chuvas e Enchentes Ocorridas em Junho de 2010 - Nota Técnica. [S.1.]: Agência Pernambucana de Água e Clima, 2010.

USGS. Flood Inundation Mapping Science. Reston, Virginia: USGS, 2012. 